Retos, 9(18), 2019

\title{
Criptomonedas y blockchain en el turismo como estrategia para reducir la pobreza
}

\section{Cryptocurrencies and blockchain in tourism as a strategy to reduce poverty}

Dr. Israel Barrutia Barreto es profesor e investigador de la Universidad Científica del Sur (Perú) (ibarrutia@ucientífica.edu.pe) (https://orcid.org/0000-0002-5728-0651)

Dr. José Antonio Urquizo Maggia es profesor e investigador de la Universidad Nacional Federico Villarreal (Perú) (josurisc@ gmail.com) (https://orcid.org/0000-0002-6999-4643)

Dr. Samuel Isaias Acevedo es profesor y director técnico de investigaciones en Innova Scientific SAC (Perú) (sacevedo@innovascientific.edu.pe) (https://orcid.org/0000-0002-1505-6117)

\section{Resumen}

La pobreza en América Latina y el Caribe sigue siendo un problema sin aparente solución. Se propone en este artículo el uso de las criptomonedas y la tecnología de blockchain como una herramienta para reducir la pobreza en la región mediante actividades económicas provenientes del turismo. Para ello se efectuó una revisión y análisis detallado de las potencialidades que recogen en conjunto el turismo, las criptomonedas y la tecnología blockchain. Dada las capacidades turísticas de la región latinoamericana y caribeña, se debe concretar un conjunto de esfuerzos por parte de los gobiernos y empresas privadas en implementar el desarrollo turístico en regiones y localidades con gran biodiversidad y recursos naturales y culturales aún sin explorar. La relativa facilidad de acceso a cuentas bitcoin mediante teléfonos inteligentes hace que las transacciones financieras mediante criptomonedas se encuentran al alcance pequeños comerciantes que, normalmente, no tienen acceso a cuentas bancarias tradicionales. Por otro lado, debe fortalecerse aún más el acceso a internet vía telefonía móvil para facilitar los sistemas de pago y para que las tecnologías basadas en blockchain puedan desarrollarse a su máxima capacidad. Se concluye que para lograr una significativa reducción de la pobreza es necesario la confluencia de una adecuada regulación de las criptomonedas por parte de los Gobiernos, así como también el desarrollo una infraestructura adecuada que permita la creación y/o recuperación de microempresas potenciadas por la «Oferta Inicial de Monedas».

\begin{abstract}
Poverty in Latin America and the Caribbean continues to be a problem with no apparent solution. We propose in this article the use of cryptocurrencies and blockchain technology as a tool to reduce poverty in the region through economic activities from tourism. A detailed analysis of the potentialities collected by tourism, cryptocurrencies and blockchain technology was carried out. Given the tourism capabilities of the Latin American and Caribbean regions, a set of efforts must be made by governments and private companies to implement tourism development in regions and locations with great biodiversity and cultural resources not yet explored. The relative access to bitcoin accounts through smartphones means that financial transactions through cryptocurrencies are available to small merchants who normally do not have access to traditional bank accounts. On the other hand, access to the internet via mobile telephony must be further strengthened to facilitate payment systems and so that blockchain-based technologies can be developed at maximum capacity. It is concluded that in order to achieve a significant reduction of poverty it is necessary the confluence of an adequate regulation of the cryptocurrencies by the governments as well as the development of an adequate infrastructure that allows the creation and / or recovery of microenterprises boosted by the "Offer Initial of Coins» (ICO).
\end{abstract}

\section{Palabras clave I keywords}

Economía del turismo, turismo y desarrollo, crecimiento económico de la economía abierta, medición y análisis de la pobreza, bienestar y pobreza.

Tourism economics, tourism and development, economic growth of open economics, measurement and analysis of poverty, welfare and poverty.

Cómo citar: Barrutia Barreto, I., Urquizo Marggia, J.A. y Acevedo, S.I. (2019). Criptomonedas y blockchain en el turismo como estrategia para reducir la pobreza. Retos Revista de Ciencias de la Administración y Economía, 9(18), 287-302. https://doi.org/ 10.17163/ret.n18.2019.07 


\section{Introducción}

A raíz de la crisis económico financiera de 2008, originada por problemas en las hipotecas en Estados Unidos que a su vez trajo una crisis alimentaria global, surgió una desconfianza generalizada en el sistema financiero mundial. De esta desconfianza se erige una propuesta por parte de Satoshi Nakamoto en el 2008 de: "una fuente alternativa de dinero, basada en una moneda virtual que permita a todas las personas enviar o recibir pagos de una forma descentralizada del sistema financiero tradicional" (Nakamoto, 2008, p.3). El uso de las criptomonedas ha tenido un creciente interés por parte de gobiernos, bancos, empresas, ámbitos académicos entre otros. El éxito se debe a que están basadas en una tecnología denominada blockchain, la cual según Nakamoto se define como:

Un libro contable compartido por los usuarios a través de una red peer to peer [red de ordenadores conectados entre sí para intercambiar información sin necesidad de un servidor fijo] que se actualiza constantemente y en el que todas las transacciones quedan registradas, pero no se comparte en ningún momento información personal (Nakamoto, 2008, p. 6).

En 2001, los líderes en las diversas sesiones de las conferencias internacionales en la Organización Internacional del Trabajo (OIT) han reafirmado el compromiso para erradicar la pobreza y su obligación de extender [...] "medidas de seguridad social para proporcionar un ingreso básico a todos los que necesitan tal protección y atención médica integral" (OIT, 2001, p. 46). La Comisión Económica para América Latina y el Caribe (CEPAL), en su «Agenda 2030», enfatiza la importancia de la protección social como clave bajo el enfoque de poner fin a la pobreza en todas sus formas en todas partes (CEPAL, 2019).

Uno de los mecanismos para reducir la pobreza es el desarrollo del turismo a escala rural y regional (Goh, 2015; CEPAL, 2018). Esta relación entre la reducción de la pobreza y el desarrollo del turismo fue destacada por la Comisión de Desarrollo Sostenible de las Naciones Unidas (CSD) en 1999 donde se acordó: "maximizar el potencial del turismo para erradicar la pobreza mediante el desarrollo de estrategias apropiadas en cooperación con todos los grupos principales y comunidades indígenas y locales" (CSD, 1999). Sin embargo, la pobreza actualmente es un concepto multidimensional, cuyo significado y medición han cambiado a lo largo del tiempo.

Algunos autores promueven efectuar investigaciones en el turismo desde la óptica de las criptomonedas y encontrar potenciales oportunidades para el desarrollo (Önder \& Treiblmaier, 2018). Con algoritmos como administradores de la escasez, las criptomonedas se convierten en el «oro del futuro»: un refugio seguro para todos los que desconfían de la rueda y el trato del stablishment (Dierksmeier \& Seele, 2018).

El desarrollo del turismo en pro o a favor de la reducción de la pobreza continúa siendo investigado con gran interés y cada vez más está atrayendo atención en el mundo académico (Yu, Wang \& Marcouiller, 2019). Estudios revelan que el turismo puede provocar el desarrollo y la reducción de la pobreza mediante tres efectos: efectos directos (trabajos creados por hoteles y restaurantes), efectos secundarios (servicios de limpieza y servicios prestados a hoteles y restaurantes) y efectos dinámicos (Croes \& Rivera, 2016; Den Braber, Evans \& Oldekop, 2018). Los efectos dinámicos 
están relacionados con el impacto general del turismo en la economía, yendo más allá del sector turístico.

Parte del problema es que la capacidad del turismo para ayudar a los pobres depende de las condiciones contextuales, como la combinación de incentivos y capacidades organizativas (Tumusiime \& Vedeld, 2012). Esta combinación de incentivos y capacidades organizativas definen la manera en la que se estructuran las economías turísticas; de modo que el impacto del turismo en los pobres depende más de la estructura de las economías turísticas y menos de los tipos de turistas que patrocinan destinos. Estas imperfecciones se derivan de la falta de confianza tan generalizada en la creación de la experiencia turística (Thomas \& Koens, 2016), de modo que la relación entre el desarrollo del turismo y la reducción de la pobreza no es evidente (Li, Jin \& Shi, 2018).

Dada la complejidad que implica la reducción de la pobreza en el contexto turistico ¿puede la implementación de las criptomonedas y la tecnología blockchain ser una herramienta para la reducción de la pobreza en América Latina y el Caribe mediante las actividades económicas provenientes del turismo?

En este caso, el rol de esta tecnología como mediador entre el turismo y la pobreza debe ser estudiado a profundidad. Por esta razón, se propone en esta investigación identificar mediante una revisión de literatura las ventajas y las potencialidades que las criptomonedas y la tecnología blockchain pueden brindar en el contexto del desarrollo turístico latinoamericano y caribeño para reducir la pobreza. En este sentido se efectuó una revisión de artículos en revistas indexadas en Scopus y Web of Science (WoS), mediante búsqueda por palabras clave y pares de palabras clave tales como Turismo-Criptomonedas, Turismo-Blockchain, Turismo-Pobreza entre otros. En este proceso se obtuvo una lista de 125 publicaciones que se consideraron dentro del alcance requerido para este estudio. De estas 125 publicaciones fueron seleccionados 62 , cuyos resultados eran más acordes al objetivo planteado en esta investigación examinando la contribución a la literatura y las limitaciones de la investigación. Cada uno de los temas clave enumerados se identificó basándose en un análisis detallado del contenido y los aspectos comunes entre los estudios seleccionados. Cabe destacar que el $70 \%$ de las publicaciones referidas al tema de criptomonedas y blockchain correspondieron a los aspectos técnicos y tecnológicos, mientras que el 30\% de las publicaciones corresponden al ámbito de las aplicaciones de la tecnología blockchain a la economía y las finanzas.

\section{Aspectos relevantes de las criptomonedas y la tecnología blockchain para el desarrollo del turismo}

\subsection{La potencialidad de las criptomonedas como herramienta para in- tercambio de bienes y servicios en el marco del turismo}

Las investigaciones relativas a las aplicaciones del Bitcoin y otras criptomonedas han recibido una atención creciente en la comunidad científica. El número de documentos publicados en este tema ha aumentado a una tasa anual del 124\% (Merediz-Solà \& Bariviera, 2019). Una criptomoneda es un activo digital diseñado para funcionar como 
un medio de intercambio que utiliza la criptografía para asegurar su transacción, controlar la creación de unidades adicionales y para verificar la transferencia de activos.

El Bitcoin, creado en 2009, fue la primera criptomoneda descentralizada, luego se formaron otras con frecuencia llamadas altcoins. Bitcoin y sus derivados utilizan el control descentralizado y se oponen a los sistemas centralizados de dinero electrónico y bancos centrales. El control descentralizado está relacionado con el uso de la base de datos de transacciones de blockchain en el papel de un libro mayor distribuido.

En ese sentido, y en relación a la asociación del recurso monetario de la modernidad, debe entenderse el Bitcoin como un sistema de pago electrónico que se basa en una combinación de criptografía y una gran colección anónima y descentralizada de participantes, llamados «mineros», para verificar transacciones, sin la necesidad de cualquier tercero de confianza. Los detalles básicos, ligeramente simplificados, son los siguientes: Un propietario de Bitcoin puede enviar divisas a otro usuario utilizando una combinación de (i) su propia dirección pública (una cadena alfanumérica, algo análoga a un número de cuenta), (ii) su propia cuenta privada clave (es decir, contraseña asociada con esa dirección), y (iii) la dirección pública del destinatario para crear una transacción con una firma criptográficamente segura.

En el caso particular del Bitcoin, este puede servir como una herramienta de cobertura contra la incertidumbre del mercado y los riesgos geopoliticos (Demir, 2018; Aysan et al., 2019). Un número creciente de minoristas ahora acepta criptomonedas como método de pago. Además, cada vez más inversores intentan capitalizar la volatilidad de los precios de la moneda virtual comprándola y vendiéndola como una inversión especulativa (Shen et al., 2019; Panagioditis et al., 2018).

Reconociendo el potencial de este creciente mercado de usuarios, la inversión en negocios de moneda virtual y nuevas empresas también ha aumentado. Incluso las instituciones financieras tradicionales y la Bolsa de Nueva York han invertido, participando en una ronda de financiación para Coinbase que recaudó \$75 millones de dólares (Tu, 2018). Otra experiencia positiva en esta dirección la tiene el turismo en Grecia, el cual ofrece servicios de hotel, vuelos y precios especiales para viajeros frecuentes en el que se puede pagar con Bitcoin (Pilkington et al., 2018). En contraste, otros autores afirman que la volatilidad del Bitcoin conduce al crecimiento de la inflación y la depreciación de la moneda (Narayan et al., 2018).

Una de las grandes ventajas del uso de las criptomonedas es que, para efectuar transacciones, la tecnología necesaria es accesible y relativamente económica. El acceso a Internet mediante teléfonos móviles en la mayoría de los casos es suficiente para una transacción con criptomonedas. Esta facilidad de intercambio permite la recepción de micropagos o préstamos por aquellos que de otra manera no tendrían acceso a la liquidez ofreciendo a las personas sin servicios bancarios una oportunidad de crecimiento económico. Un estudio realizado en China, India, Nigeria y Sudáfrica muestra que los países con niveles más altos de criptomonedas, Internet y suscripciones móviles tienen también niveles más altos de inclusión financiera y desarrollo del sector financiero (Vincent \& Evans, 2019). En el caso de América Latina, más del $50 \%$ de la población se encuentra conectada a internet mediante servicios de telefonía móvil (ver Gráfico 1). 


\section{Gráfico 1. Porcentaje de poblaciones conectadas y no conectadas a servicios de Internet móvil en América Latina (2014-2017)}

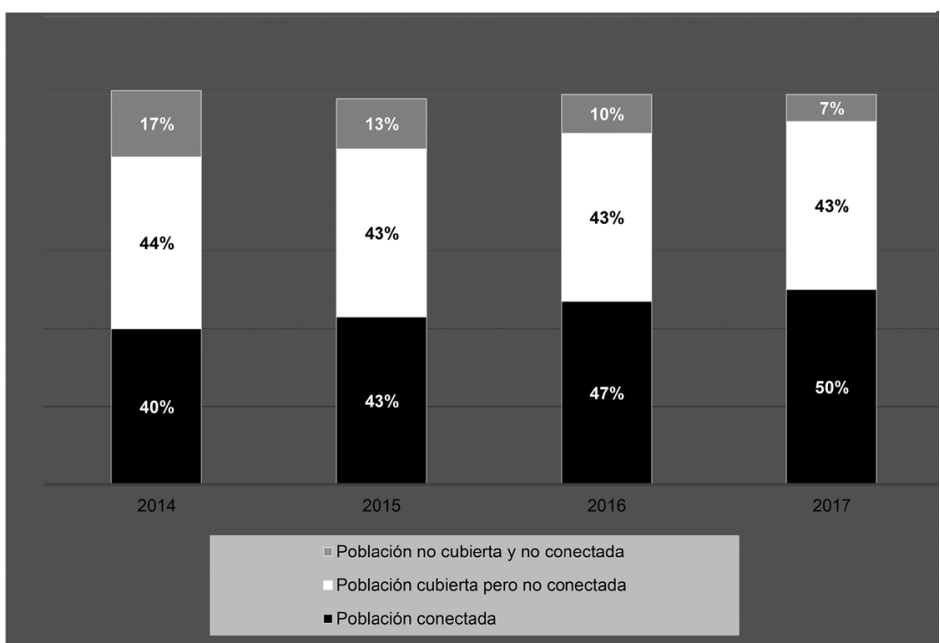

Fuente: GSMA Inteligence (2018, p. 38).

Una de las formas tradicionales para salir de la pobreza es accediendo a un tipo de microcrédito bancario. El microcrédito es el sistema establecido para proporcionar a las personas empobrecidas pequeños préstamos con los que puedan iniciar algunas pequeñas empresas. El microcrédito ha sido utilizado como un arma importante contra la pobreza (v.gr. Ikeda y Hamid, 2018; Ikeda y Hamid, 2018). Hasta 2009, aproximadamente 74 millones de personas tenían un microcrédito total de 38 mil millones de dólares. Ahora, también existen muchas personas que no tienen acceso a un microcrédito por no cumplir los suficientes requisitos para su aprobación. Al no tener acceso a un banco físico o la documentación requerida para el acceso a microcréditos, tales individuos no tienen, hasta el advenimiento de las criptomonedas, opciones para ahorrar y asegurar activos o de poder participar en la planificación e inversión financiera.

Debido a su dependencia del efectivo, a menudo se ponen en peligro y siempre se limitan a realizar transacciones con personas que están a su alcance físico. Más de 2.500 millones de adultos en todo el mundo carecen de una cuenta en el sector bancario formal. Según el Banco Mundial, en algunas economías, como Camboya, la República Democrática del Congo, Guinea, la República Kirguisa, Turkmenistán y la República de Yemen, más del 95\% de los adultos no tienen una cuenta en una institución financiera formal. De los 600 millones de habitantes del sudeste asiático, solo el $27 \%$ tiene una cuenta bancaria. Esta proporción es tan baja como el 5\% en Camboya (Kshetri, 2017a). La investigación en financiamiento para el desarrollo sugiere que el financiamiento accesible y oportuno tiene el potencial de sacar a los pobres de su situación de pobreza (Mushtaq \& Bruneau, 2019). 
La inclusión de sistemas de pago mediante dinero móvil se está expandiendo rápidamente en los países en desarrollo. En Kenia se encontró que el uso de los teléfonos móviles puede reducir significativamente las dificultades de las comunidades desfavorecidas para acceder a servicios sociales específicos (Lashitew, Van Tulder \& Liasse, 2019). Otro estudio reciente realizado en Ghana demuestra que tener acceso a un teléfono móvil mejora las posibilidades de no ser pobre y puede acelerar significativamente los ingresos de pequeñas empresas (Danquah \& Iddrisu, 2018). Asimismo, en zonas rurales de India se encontró que la inclusión rápida de teléfonos móviles crea una oportunidad para mejorar el acceso de las personas a la atención médica (Haenssgen, 2018).

Con la incorporación de las criptomonedas se puede lograr que los costos de las transacciones sean mucho menores. Debido a la creciente popularidad del uso de la criptomoneda como un nuevo medio de pago, muchas empresas están tomando una nueva forma de lanzar sus propias criptomonedas, llamada «Oferta Inicial de Monedas» (ICO, por su acrónimo en inglés), el cual se define como un: "sistema de inversión o financiación que utilizan las empresas basadas en blockchain con la finalidad de obtener capital y darle valor a los tokens o monedas que son usadas en sus aplicaciones o en los diferentes servicios que ofertan" (García, 2018, p. 22). En vez de ofertarse acciones, como es el caso de una Oferta Pública de Venta (OPV), se hace una venta de «tokens» que pueden ser cambiados por monedas fiduciarias, por monedas virtuales o por servicios que la propia empresa ofrezca Es una forma bastante nueva, no estandarizada, de recaudar fondos iniciales que puede brindar altos rendimientos a los inversores a corto plazo (Lahajnar \& Rožanec, 2018).

Una ICO podría describirse simplemente como un evento en el que una startup o proyecto vende su nueva criptomoneda por primera vez al público para recaudar capital (Dhillon, Metcalf \& Hooper, 2017; Hill, 2018; Chen, 2018). El equipo del proyecto que inicia una ICO determinará el precio de venta inicial. Dependiendo de la naturaleza del proyecto, los inversores podrían usar estas criptomonedas como monedas o «tokens» que podrían interactuar con el producto futuro. Desde entonces, el precio de la criptomoneda específica se liquidará a través de la oferta y la demanda en el mercado (Kastelein, 2017).

Tanto la cantidad de ICO, como la cantidad de capital recaudado, han explotado desde 2017 (Fish, 2019). Muchos de los proyectos de apalancamiento basados en ICO tienen una tasa de éxito bastante alta. Mientras que los fondos recaudados mediante capital de riesgo tradicional en el 2017 fueron de 350 millones de dólares, mediante la financiación de empresas blockchain el volumen de emisión de ICO alcanzó los 2 mil millones de dólares en el mismo periodo (Nolan et al., 2018). El mercado secundario para «tokens» ICO es bastante líquido en el primer día de negociación y el rendimiento inicial es grande, alrededor de un valor medio de $+919.9 \%$ comparado con el precio de oferta cuyo valor medio es de $+24.7 \%$ (Adhami, Giudici \& Martinazzi, 2018).

Sobre la base de entrevistas de varios expertos en el campo de la criptografía se han podido identificar algunas señales importantes para invertir en el proceso de ICO: la relación del medio ambiente local (gobierno) para invertir en proyectos de tecnología blockchain, historia de la compañía, liquidez emitida en «tokens» y su distribución, respuesta de comunidades criptográficas en el proyecto, bonos promocionales, anuncios 
pagados y la calidad de la información (Yadav, 2017). Esto es de vital importancia a la hora de implementar un nuevo emprendimiento en el contexto turístico.

\subsection{Ventajas y limitaciones del desarrollo del turismo en los países de América Latina y el Caribe}

El turismo es relativamente accesible a los pobres, pues a través de él se pueden generar fuentes de empleo a mujeres, jóvenes y grupos desfavorecidos como minorías étnicas. Además, la actividad turística puede potenciar la agricultura, el transporte y la artesanía (Njoya \& Seetaram, 2017; Li et al., 2018), por lo que es meridianamente evidente que el turismo podría proporcionar beneficios a corto plazo a los pobres. Sin embargo, estos beneficios podrían disminuir en un largo plazo (Sharpley \& Naidoo, 2010; Zeng et al., 2015).

En Estados insulares en desarrollo, el turismo contribuye a la reducción de la pobreza y puede mejorar el desarrollo humano (Jiang et al., 2011; Kim, Uysal \& Sirgy, 2013), mientras que, en las áreas rurales remotas, el empleo en ecoturismo mejora la seguridad financiera y el bienestar social (Snyman, 2012; Chirenje, 2017), aunque preexisten factores socioculturales que pueden limitar la participación de hombres y mujeres en el turismo (Tucker \& Boonabaana, 2012). Por un lado, el turismo proporciona una diversificación de los medios de vida para los locales, y por el otro, aquellos que dependían del empleo en el sector de alojamientos tenían los ingresos más bajos (Adiyia et al., 2014).

Por su parte, se ha reconocido el impacto que puede tener el turismo en el PIB. En 2018, la región Asia-Pacífico creció un 6,4\%, en el norte de África se registró un crecimiento anual del 8,6\%. Similarmente Etiopía, Ecuador, San Cristóbal y Nieves, Egipto y Turquía lideraron el crecimiento mundial del PIB en viajes y turismo en 2018 (World Travel \& Tourism Council, 2019). El turismo hace aportes importantes al Producto Interno Bruto de los países, teniendo en el caso Latinoamérica un crecimiento importante (ver Gráfico 2).

\section{Gráfico 2. Contribución directa en miles de millones de USD del sector de viajes y turimo al Producto Interno Bruto (PIB) en América Latina (2012 y el 2018)}

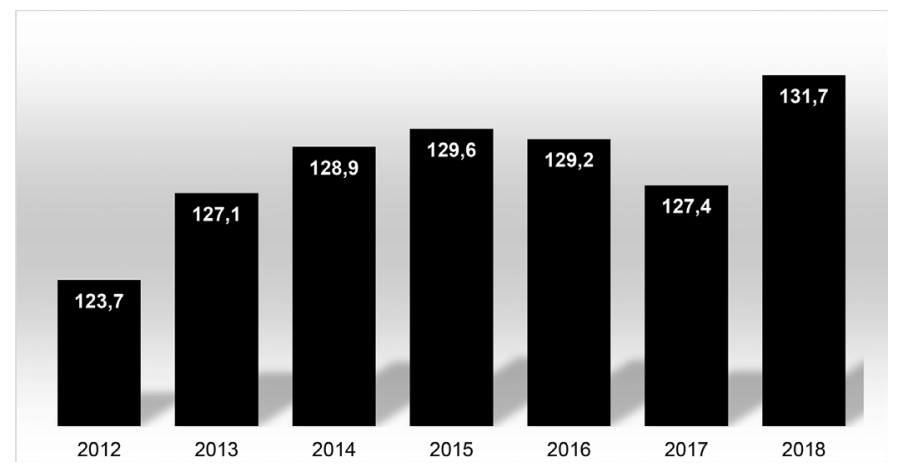

Fuente: Elaboración propia, con datos tomados del World Travel \& Tourism (2019, p. 8). 
A pesar de las ventajas de los ingresos del turismo, algunos afirman que dichos ingresos no reducen la pobreza sino que afianzan la desigualdad (Mahadevan \& Suardi, 2019). En Centroamérica las elasticidades de la pobreza variaron, teniendo efectos fuertes en Costa Rica, Guatemala y Nicaragua y efectos moderados a débiles en Salvador y Honduras (MacNeill \& Wozniak, 2018). En China el turismo es beneficicioso cuando los servicios de alojamiento y restaurantes son construidos por los mismos residentes locales mientras que, al hacerse inversiones de capital por parte de empresas privadas, los habitantes locales no resultaban beneficiados con el crecimiento del sector (Lor, Kwa \& Donaldson, 2019).

En muchos casos se ha sugerido que el turismo alivia la pobreza siempre y cuando se generen programas de inclusión social (Medina-Muñoz, Medina-Muñoz \& Gutiérrez-Pérez, 2016a). En Kenya, se encontró que el turismo reduce significativamente la brecha entre la pobreza y la pobreza extrema, con mayor impacto en las áreas urbanas que en las áreas rurales (Njoya \& Seetaram, 2017). Similarmente, otros consideran que el turismo corporativo impacta en la disminución de la pobreza local, regional y nacional (Medina-Muñoz, Medina-Muñoz \& Gutiérrez-Pérez, 2016b). Se han dado esfuerzos por empoderar a las comunidades para que sean protagonistas en el desarrollo turístico en su región como es el caso de Perú, aunque los resultados concretos no han sido cuantificados en su totalidad (Knight \& Cotterll, 2016).

Recientes investigaciones sugieren que para cambiar los aspectos negativos del turismo y hacerlo más equitativo y sostenible se debe cambiar el enfoque de fomentar el crecimiento económico a considerar los aspectos sociopolíticos de la pobreza y cómo las desigualdades estructurales están impidiendo el desarrollo de las personas (Scheyvens \& Hughes, 2018; Truong, 2018).

Por su parte, también se han desarrollado estudios que consideran los efectos de largo plazo que pueda tener el cambio climático en los patrones de flujo turístico (Bujosa, Riera \& Torres, 2015), de modo que para el desarrollo de un turismo sostenible se deben evaluar las vulnerabilidades que implican los factores climáticos, económicos, sociales y políticos del entorno (Dogru, Marchio, Bulut \& Suess, 2019).

En sentido general, se evidencia un contraste en cuanto al impacto que puede tener el turismo en la disminución de la pobreza. El turismo en América Latina y el Caribe se encuentra todavía en una fase de desarrollo embrionario, convirtiéndolo en una gran oportunidad de inversión y crecimiento, sabiendo las potencialidades y ventajas que ofrece los abundantes recursos naturales que caracterizan a la región.

\subsection{El rol de la tecnología Blockchain en la gestión de la cadena suminis- tro, logística y contratos inteligentes}

Los temas mas discutidos en la literatura en donde la tecnología blockchain puede generar cambios significativos giran en torno a contratos inteligentes, gestión de la cadena de suministro y logística. La tecnología blockchain es considerada como la mayor innovación en ciencias de la computación, así como el mayor disruptor de las industrias desde la introducción de Internet (Kshetri, 2017b). Las características tecnológicas del blockchain se prestan para el procesamiento de contratos inteligentes donde los pagos de liquidación y los pagos financieros pueden activarse automáticamente por eventos específicos. El estudio de Iansiti y Lakhani (2017) analiza un escenario de un 
contrato inteligente en el que se utiliza la tecnología blockchain para automatizar un pago a un proveedor tan pronto como se recibe el envío a través del GPS rastreando las mercancías, monitoreando la entrega para desencadenar el pago del proveedor (Iansiti \& Lakhani, 2017).

El proceso tradicional de la cadena de suministro se basa en intermediarios externos que actúan como garantes de los activos y mecanismo de aseguramiento de la propiedad de los bienes. Al implementar soluciones basadas en blockchain, todas las partes pueden determinar automáticamente la propiedad de los bienes, aprobar el envío y desencadenar los pagos al recibirlos (Dobrovnik et al., 2018). Las organizaciones están revisando sus procesos y modelos de negocios que buscan identificar los casos de uso clave donde blockchain puede agregar valor y brindar beneficios (Ying, Jia \& Du, 2018; Zamani \& Giaglis, 2018).

El potencial de la tecnología del blockchain ya ha sido reconocida por el sector financiero y grandes empresas como IBM, Deutsche Bank, HSBC, Société Générale y UniCredit, solo por nombrar algunas, están construyendo sistemas para facilitar el comercio de pequeñas y medianas compañías (Önder \& Treiblmaier, 2018). Una reducción en los costos de transacción para pagos pequeños puede ser tan importante como para decidir el éxito o el fracaso de un modelo de negocios. Mediante blockchain se pueden reducir los costos de transacción para todos los modelos híbridos de empresas sociales, cuya sostenibilidad financiera se basa en parte en donaciones o apoyo público. Adoptando tecnologías innovadoras basadas en blockchain, es posible además reducir los costos de la recaudación de capital, evitando intermediarios y agentes de pago.

Para el caso de los medios de financiamientos convencionales, cada año, más de mil millones de dólares fluyen de individuos, gobiernos y empresas, para abordar los desafíos de la pobreza y la crisis en todo el mundo (Carraro, 2017). La distribución y el seguimiento de los fondos para el desarrollo mundial y la ayuda humanitaria siguen siendo complejos, opacos y enormemente ineficientes (Banco Mundial, 2017). La falta de transparencia también es un tema clave, ya que existe una incapacidad para rastrear el flujo de fondos de un extremo a otro. La ONU estima que hasta el $30 \%$ de la asistencia oficial para el desarrollo se pierde debido al fraude y la corrupción. En el terreno, las organizaciones enfrentan múltiples barreras para garantizar la transparencia total de la distribución de fondos (Thomason et al., 2018).

Con relación a la reducción de costos que implica el uso de las tecnologías blockchain, la diferencia es significativa con relación a las formas de pago convencionales si se trata de intercambios pequeños como donaciones o microcréditos para agricultores con orientación al turismo para países en desarrollo (Dierksmeier y Seele, 2018). La tecnología blockchain tiene el potencial de contribuir a una serie de Objetivos de Desarrollo Sostenible de la ONU (Hughes et al., 2019).

Proyectos de factibilidad desarrollados por Walmart e IBM para usar la tecnología blockchain para monitorear específicamente productos basados en alimentos en los Estados Unidos y China ayudaron a reducir significativamente el tiempo necesario para hacer un seguimiento de los alimentos de días a minutos (Kshetri, 2018a). El grupo Hainan Airlines (HNA) en China desarrolló un sistema de comercio electrónico 
basado en blockchain para que sus empleados compren productos directamente de proveedores externos. El sistema se puso en línea en febrero de 2015 con 2000 proveedores participantes y se consideró una implementación exitosa (Ying, Jia \& Du, 2018). La tecnología blockchain es prometedora y una correcta implementación en el ámbito del turismo podría contribuir de forma más efectiva a la disminución de la pobreza.

\subsection{Estatus legal de las criptomonedas y la tecnología blockchain}

Los gobiernos, aunque tarde en reconocer la importancia del Bitcoin, ahora están considerando tanto la regulación como los impuestos a esta criptomoneda (Holub \& Johnson, 2018). En muchos escenarios, la regulación de Bitcoin no es visto como un problema apremiante para los gobiernos, sino más bien el hecho de que una regulación severa basada en los peores escenarios podría sofocar fácilmente las oportunidades que ofrece tal innovación (De Filipi \& Loveluck, 2014). En términos generales, la discusión e implementación de un marco legal apropiado está relativamente poco desarrollada (Tu, 2018). Igual sucede en el caso de la tecnología blockchain, aunque las organizaciones parecen estar revisando activamente el blockchain a nivel estratégico, los enormes desafíos en las regulaciones destacan la inmadurez de la tecnología (Lacity, 2018). La mayoría de los países en el mundo no han adoptado un marco legal sobre el uso de las criptomonedas y blockchain, sin embargo, ya se han hecho bastantes adelantos.

En Rusia, el gobierno ha reconocido que una prohibición absoluta de la criptomoneda no traería resultados positivos y están buscando su propio enfoque de la regulación legal de ésta. En el caso de la tecnología blockchain, ha comenzado a usarse como un sistema para registrar transacciones de bienes y raíces (Zharova \& Lloyd, 2018). Japón es otro caso donde se ha venido aceptando criptomonedas como medio de intercambio. En el ámbito de la tecnología blockchain se está trabajando en unificar todo el registro de propiedades urbanas y turísticas. En Vietnam, por ejemplo, el Estado prohíbe el uso de las criptomonedas y penaliza con multas de hasta US $\$ 8800$ (García, 2018). En el caso de Europa, Bitcoin ya ha sido aceptado por el Tribunal de Justicia Europeo como moneda válida y está exento de impuestos sobre las ventas (Önder \& Treiblmaire, 2018). Alemania reconoce el Bitcoin como instrumento financiero y se puede utilizar para fines comerciales y fiscales. En España, las compras y ventas de criptomonedas son reconocidas como actividades económicas y están exentas de IVA.

Para el caso de los países de América Latina y el Caribe, la mayoría no posee un marco regulatorio que incentive las actividades con criptomonedas y blockchain (ver Gráfico 3). Incluso, en países como Bolivia y Ecuador se prohíben expresamente las actividades financieras con Bitcoin. 


\section{Gráfico 3. Estatus legal sobre el uso de las criptomonedas en América Latina y el Caribe durante el año 2018 (\%)}

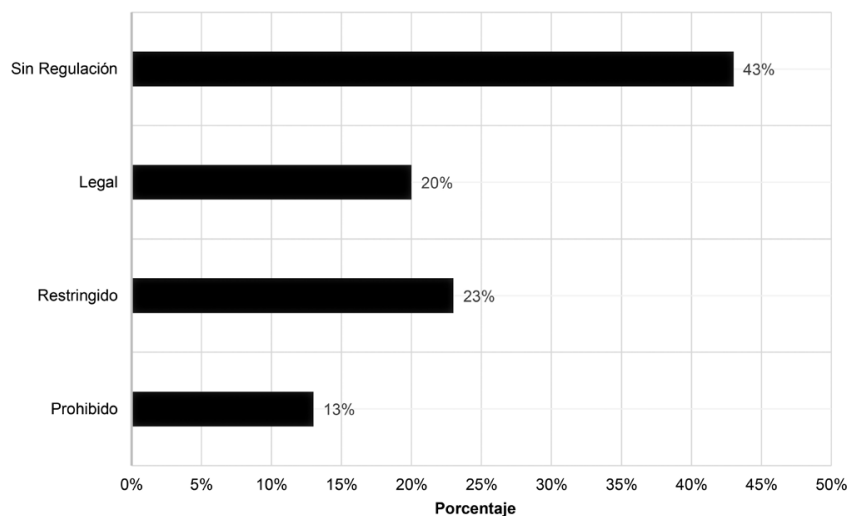

Fuente: Elaboración propia, con base en datos de la Library of Congress (2018, pp.1).

\section{Discusión y conclusiones}

En el contexto latinoamericano y caribeño, el turismo posee enormes potencialidades y su grado de impacto en el PIB puede incrementarse más ya que, este rubro económico sigue en fase embrionaria de desarrollo. Con base en los Objetivos de Desarrollo Sostenible (ODS) planteados por la ONU, la implementación de la tecnología blockchain y las criptomonedas en el marco del turismo pueden contribuir a disminuir la pobreza en Latinoamérica. La tecnología blockchain podría realizar un cambio significativo en los aspectos de sostenibilidad que afectan la salud, la distribución de medicamentos y los suministros humanitarios.

Existen problemas en la integridad de los medicamentos y productos alimenticios básicos donde el desafío de la aplicación y la gestión logística a través de las barreras lingüísticas y la diversidad geográfica es un gran desafío. Los atributos del blockchain podrían ofrecer beneficios en los países de América Latina y el Caribe donde la tecnología podría utilizarse para garantizar la calidad y el compromiso con la educación en todas sus formas. Los compromisos financieros y logísticos con la educación y la igualdad de género de las autoridades centrales y regionales se incluirían en los contratos inteligentes entre todas las partes interesadas. Los contratos relacionados con el desarrollo de la infraestructura necesaria para el desarrollo del turismo para garantizar agua limpia, saneamiento y energía podrían gestionarse a través de la tecnología blockchain, lo que garantiza que se minimizaría el fraude y se desarrollarían niveles más altos de confianza entre las partes. La naturaleza inmutable de la cadena de bloques y la transparencia de las transacciones pueden generar protección salarial y reducir la explotación entre los trabajadores del turismo.

Dadas las enormes capacidades turísticas de las regiones latinoamericana y caribeña se debe concretar un conjunto de esfuerzos por parte de los gobiernos y empresas privadas en implementar el desarrollo turístico en regiones y localidades 
con gran biodiversidad y recursos naturales y culturales aún sin explorar. Para ello, los gobiernos deben incentivar el desarrollo de microcréditos a pequeños y medianos empresarios del turismo mediante criptomonedas, aprovechando que las comunicaciones y el acceso a Internet en Latinoamérica y el Caribe está relativamente cubierta. Así se apalancaría el empoderamiento de las comunidades a que sean protagonistas en la creación de empresas impulsadas por ICO, con la debida regulación y apoyo de los gobiernos y las empresas privadas.

Por otro lado, debe fortalecerse aún más el acceso a Internet vía telefonía móvil para facilitar los sistemas de pago y para que las tecnologías basadas en blockchain puedan desarrollarse a su máxima capacidad. De este modo, el camino queda preparado para instalar toda una red de sistemas de pagos en Bitcoins y otras criptomonedas que permitan pagar bienes y servicios, intercambiar por otras monedas fiduciarias y construir toda una red de cajeros ATM de criptomonedas, cuya implementación ya ha sido patente en otros países.

De esta manera, aprovechando que el volumen de transacciones con criptomonedas se incrementa, se puede planificar y ejecutar esos paquetes turísticos con métodos de pago con criptomonedas y que además toda la gestión del viaje: hoteles, transporte, alimentación entre otros sea manejado por tecnologías blockchain. Para esto es necesario que el Estado promueva, acelere y facilite una normativa legal para el uso de las criptomonedas, lo cual trae un incremento en la confianza por parte de los inversionistas.

Asimismo, incentivar y educar a las comunidades en la creación de micros y medianas empresas de carácter turístico cuya logística sea manejada por tecnología blockchain, utilizando fuentes de financiamiento a través de ICO, en el que se ofrezcan «tokens» que puedan ser intercambiados por otras monedas fiduciarias, por otras criptomonedas y otros bienes y servicios que beneficien tanto a la comunidad como al propio turista e inversionista. Surgen nuevas oportunidades a medida que los costos de transacción y las instituciones intermediarias desaparecen. Dada la enorme cantidad de dinero que se invierte actualmente en soluciones basadas en blockchain y su naturaleza que abarca la industria, se puede esperar que el impacto en el sector empresarial especialmente en la industria del sector turístico sea sustancial y traer como consecuencia un mejoramiento en las condiciones de vida en las poblaciones más desfavorecidas.

La investigación sobre el efecto de la tecnología blockchain y las criptomonedas en el turismo en la literatura es escasa, por lo que se sugiere investigar más en el tema desde una perspectiva correlacional. Al ser el mercado turístico en Latinoamérica de carácter emergente, se puede implementar la tecnología blockchain y las criptomondeas con menos dificultad que en aquellos países donde el turismo ya está desarrollado. La investigación en estudios de casos exitosos en otras latitudes puede arrojar luz para replicar la experiencia en Latinoamérica y el Caribe. Mediante políticas gubernamentales debe realizarse un diagnóstico del potencial turístico y acoplar con la empresa privada una adaptación de la economía basado en criptomonedas para el acceso a servicios de salud, educación y oportunidades mediante el uso de la tecnología móvil. De este modo, las poblaciones mas desfavorecidas pueden incrementar los ingresos al establecerse precios justos en el intercambio de bienes y servicios o en la distribución equitativa de las ganancias mediante blockchain. 


\section{Referencias}

Adhami, S., Giudici, G., \& Martinazzi, S. (2018). Why do bussinesses go crypto? An empirical analysis of Initial Coin Offerings. Journal of Economics and Business, 100, 64-75. https://doi. org/10.1016/j.jeconbus.2018.04.001

Adiyia, B., Vanneste, D., Van Rompaey, A., \& Ahebwa, W. M. (2014). Spatial analysis of tourism income distribution in the accomodation sector in western Uganda. Tourism and Hospitality Research, 14(1-28-26. https://doi.org/10.1177\%2F1467358414529434

Aysan, A. F., Demir, E. Gozgor, G., \& Marco, C. K. (2019). Effects of the geopolitical risks on Bitcoin returns and volatility. Research in International Business and Finance, 47, 511-518. https:// doi.org/10.1016/j.ribaf.2018.09.011

Bujosa, A. Riera, A., \& Torres, C. (2015). Valuing tourism demand attributes to guide climate change adaptation measures efficiently: The case of the Spanish domestic travel market. Tourism Management, 47, 233-239. https://doi.org/10.1016/j.tourman.2014.09.023

Carraro, C. (2017). Towards a greener finance landscape, A blog by Carlo Carraro, 28 de marzo de 2017. Recuperado de https://bit.ly/2KxsT3R [Fecha de consulta: 19 de febrero de 2019]

Chen, Y. (2018). Blockchain tokens and the potential democratization of entrepreneurship and innovation. Business Horizons, 61(4), 567-575. https://doi.org/10.1016/j.bushor.2018.03.006

Chirenje, L. I. (2017). Contribution to ecotourism to poverty alleviation in Nyanga, Zimbabwe. Chinesse Journal of Population Resources and Environment, 15(2), 87-92. https://doi.org/10.1080/10042857.2017.131Economía, 9(18)2

Croes, R., \& Rivera, M. (2015). The Poverty Obliteration Paradigm. En M. Khan (Series Ed.), Advances in Hospitality Series. Poverty Alleviation through Tourism Development: A Comprehensive and integrated Approach (pp. 47-74). New York, Estados Unidos: Apple Academic Press. https:// doi.org/10.1201/b19841

Danquah, M., \& Iddrisu, A. M. (2018). Access to mobile phones and the wellbeing of non-farm enterprise households: Evidence from Ghana. Technology in Society, 54, 1-9. https://doi.org/10.1016/j.techsoc.2018.01.012

De Filipi, P., \& Loveluck, B. (2016). The Invisible Politics of Bitcoin: Governance Crisis of a Descentralised Infrastructure. Internet Policy Review, 5(3), 1-28. https://doi.org/10.14763/2016.3.427

Dhillon, V., Metcalf, D., \& Hooper, M. (2018). Blockchain Enabled Applications: Understand the Blockchain Ecosystem and How to Make it Work for You. California, Estados Unidos: Apress. https://doi. org/10.1007/978-1-4842-3081-7

Dierskmeier, C. \& Seele, P. (2018). Cryptocurrencies and Business Ethics. Journal of Business Ethics, 152(1), 1-14. https://doi.org/10.1007/s10551-016-3298-0

Dobronik, M., Herold, D. M., Furst, E., \& Kummer, S. (2018). Blockchain for and in Logistics: What to Adopt and Where to Start. Logistics, 2(3), 18-32. https://doi.org/10.3390/logistics2030018

Dogru, T. Marchio, E.A., Bulut, U., \& Suess, C. (2019). Climate change: Vulnerability and resilience of tourism and the entire economy. Tourism Management, 72, 292-305. https://doi. org/10.1016/j.tourman.2018.12.010

Fisch, C. (2019). Initial coin offerings (ICOs) to finance new ventures. Journal of Business Venturing, 34(1), 1-22. https://doi.org/10.1016/j.jbusvent.2018.09.007

García, J. (2018), Criptomonedas y Aplicación en la Economía (Tesis de Maestria). Universidad Pontificia Comillas, España. Recuperado de: http://hdl.handle.net/11531/32886

Goh, H. C. (2015). Nature and Community-based tourism (CBT) for poverty alleviation: A case study of Lower Kinabatangan, East Malaysia. Geografia Malaysian Journal of Society and Space, 11(3), 42-52. Recuperado de: https://bit.ly/2GTlg6G

GSMA Intelligence (2018). La Economía móvil en América Latina y el Caribe, 03 de noviembre de 2018. Recuperado de http://bit.ly/2JnHTm8 [Fecha de consulta: 15 de febrero de 2019]

Haenssgen, M.J. (2018). The struggle for digital inclusion: Phones, healthcare and marginalization in Rural India. World Development, 104, 358-374. https://doi.org/10.1016/j.worlddev.2017.12.023 
Hill, J. (2018). FinTech and the Remaking of Financial Institutions. Estados Unidos: Academic Press. https://doi.org/10.1016/C2016-0-03863-9

Holub, M., \& Johnson J. (2019). Bitcoin research across disciplines. The Information Society, 34(2), pp. 114-126. https://doi.org/10.1080/01972243.2017.1414094

Hughes, L., Dwivedi, Y. K., Misra, S.K., Rana, N.P., Raghavan, V., \& Akella, V. (2019). Blockchain research, practice and policy: Applications, benefits, limitations, emerging research themes and research agenda. International Journal of Information Management, 49, 114-129. https:// doi.org/10.1016/j.ijinfomgt.2019.02.005

Iansiti, M., \& Lakhani, K.R. (2017). The truth about blockchain. Harvard Business Review, 95(1), 118127. Recuperado de: https://hbr.org/2017/01/the-truth-about-blockchain

Ikeda, K., \& Hamid M. (2018). Application of Blockchain in the Financial Sector and a Peerto-Peer Global Barter Web. En P. Raj y G. C. Deka (Eds.), Advances in Computers: Vol. 111. Blockchain Technology: Platforms, Tools and Use Cases (pp. 99-120). Estados Unidos: Academic Press. https://doi.org/10.1016/bs.adcom.2018.03.008

Jiang, M., DeLacy, T., Mkiramweni, N.P., \& Harrison, D. (2011). Some evidence for tourism alleviating poverty. Annals of Tourism Research, 38(3), 1181-1184. https://doi.org/10.1016/j. annals.2011.03.008

Jing, W., Jia, S., \& Du, W. (2018). Digital enablement of blockchain: Evidence from HNA group. International Journal of Information Management, 39, 1-4. https://doi.org/10.1016/j.ijinfomgt.2017.10.004

Kastelein, R. (2017). What Initial Coin Offerings Are, and Why VC Firms Care. Harvard Business Review, 24 de marzo del 2017. Recuperado de https://bit.ly/2mZgh81

Kim, K., Uysal, M., \& Sirgy, J. (2013). How does tourism in a community impact the quality of life of community residents?, Tourism Management, 36, 527-540. https://doi.org/10.1016/j. tourman.2012.09.005

Knight, D.W., \& Cottrell, S.P. (2016). Evaluating tourism-linked empowerment in Cuzco, Perú. Annals of Tourism Research, 56, 32-47. https://doi.org/10.1016/j.annals.2015.11.007

Kshetri, N. (2017a). Potential roles of blockchain in fighting poverty and reducing financial exclusion in the global south. Journal of Global Information Technology Management, 20(4), 201204. https://doi.org/10.1080/1097198X.2017.1391370

Kshetri, N. (2018a). Blockchain's roles in meeting key supply chain management objectives. International Journal of Information Management, 39, 80-89. https://doi.org/10.1016/j.ijinfomgt.2017.12.005

Kshetri, N. (2017b). Will blockchain emerge as a tool to break the poverty chain in the Global South? Third World Quarterly, 38(8), 1710-1732. https://doi.org/10.1080/01436597.2017.12 98438

Lacity, M. C. (2018). Addresing Key Challenges to Making Enterprise Blockchain Applications a Reality. MIS Quarterly Executive, 17(3), 201-222.

Lahajnar, S., \& Rožanec, A. (2018). Initial Coin Offering (ICO) Evaluation Model. Investment Management and Financial Innovations, 15(4), 169-182. http://dx.doi.org/10.21511/imfi.15(4).2018.14

Lashitew, A. A., Van Tulder, R., \& Liasse, Y. (2019). Mobile phones for financial inclusion: What explains the diffusion of mobile money innovations. Research Policy, 48(5), 1201-1215. https://doi.org/10.1016/j.respol.2018.12.010

Li, K. X., Jin, M., \& Shi, W. (2018). Tourism as an important impetus to promoting economic growth: A critical review. Tourism Management Perspectives, 26,. 135-142. https://doi.org/10.1016/j.tmp.2017.10.002

Li, T., Liu, J., Zhu, H., \& Zhang, S. (2018). Business characteristics and efficiency of rural tourism enterprises: an empirical study from China. Asia Pacific Journal of Tourism Research, 23(6), 549-559. https://doi.org/10.1080/10941665.2018.1483957

Library of Congress (2018). Regulation of Bitcoin in Selected Jurisdictions, 23 de Julio de 2018. Recuperado de http://bit.ly/2V7pk7F [Fecha de consulta: 16 de febrero de 2019] 
Lor, J.J., Kwa, S., \& Donaldson, J.A. (2019). Making ethnic tourism good for the poor. Annals for Tourism Research, 76, 140-152. https://doi.org/10.1016/j.annals.2019.03.008

MacNeill, T., \& Wozniak, D. (2018). The economic, social and environmental impacts of cruise tourism. Tourism Management, 66, 387-404. https://doi.org/10.1016/j.tourman.2017.11.002

Mahadevan, R., \& Suardi, S. (2019). Panel evidence on the impact of tourism growth on poverty, poverty gap and income inequality. Current Issues in Tourism, 22(3), 253-264. https://doi.org $/ 10.1080 / 13683500.2017 .1375901$

Medina-Muñoz, D.R., Medina-Muñoz, R.D., \& Gutiérrez-Pérez, F.J. (2016a). The impacts of tourism on poverty alleviation: an integrated research framework. Journal of Sustainable Development, 24(2),. 270-298. https://doi.org/10.1080/09669582.2015.1049611

Medina-Muñoz, D.R., Medina-Muñoz, R.D., \& Gutiérrez-Pérez, F.J. (2016b). A Sustainable Development Approach to Assessing the Engagement of Tourism Enterprises in Poverty Alleviation. Sustainable Development, 24(4), 220-236. https://doi.org/10.1002/sd.1624

Merediz-Solà, I., \& Bariviera, A.F. (2019). A bibliometric analysis of bitcoin scientific production. Research in International Business and Finance, 50, 294-305. https://doi.org/10.1016/j.ribaf.2019.06.008

Mushtaq, R., \& Bruneau, C. (2019). Microfinance, financial inclusion and ICT: Implications for poverty and inequality. Technology in Society, 59. Advance online publication. https://doi. org/10.1016/j.techsoc.2019.101154

Naciones Unidas. Comisión Económica para América Latina y el Caribe. (2019). La Agenda 2030 y los Objetivos de Desarrollo Sostenible. Una oportunidad para América Latina y el Caribe. Chile: Naciones Unidas. Recuperado de http://bit.ly/2VNVfhV

Naciones Unidas. Comisión Económica para América Latina y el Caribe (2018). Ruralidad, hambre y pobreza en América Latina y el Caribe. Chile: CEPAL-FAO. Recuperado de http://bit. ly/2POhlvm

Nakamoto, S. (2008). Bitcoin: A Peer-to-Peer Electronic Cash System, 15 abril del 2008. Recuperado de http://bit.ly/2DTCfnX [Fecha de consulta: 16 de febrero de 2019]

Narayan, P. K., Narayan, S., Rahman, R. E., \& Setiawan, I. (2018). Bitcoin Price Growth and Indonesia's Monetary System. Emerging Markets Review. 38, 364-376. https://doi.org/10.1016/j. ememar.2018.11.005

Njoya, E. T., \& Seetaram, N. (2018). Tourism Contribution to Poverty Alleviation in Kenya: A Dynamic Computable General Equilibrium Analysis. Journal of Travel Research, 57(4), 513-524. https://doi.org/10.1177/0047287517700317

Nolan, A. R., Dartley, E. T., Baker, M.B., ReVeal, J., \& Rinearson, J.E. (2018). Initial Coin Offerings: Key US Legal Considerations for ICO investors and sponsors. Journal of Investment Compliance, 19(1), 1-9. https://doi.org/10.1108/JOIC-02-2018-0016

Önder, I., \& Treiblmaier, H. (2018). Blockchain and tourism: Three research propositions. Annals of Tourim Research, 72, 180-182. https://doi.org/10.1016/j.annals.2018.03.005

Organizacion Internacional del Trabajo (2001). Resolución y conclusiones relativas a la seguridad Social. Recuperado de http://bit.ly/2E5GhtR

Panagioditis, T., Stengos, T., \& Vravosinos, O. (2018). The effects of markets, uncertainty and search intensity on bitcoin returns. International Review of Financial Analysis, 63, 220-242. https://doi.org/10.1016/j.irfa.2018.11.002

Pilkington, M., Crudu, R., \& Grant, L.G. (2017). Blockchain and bitcoin as a way to lift a country out of poverty - tourism 2.0 and e-governance in the Republic of Moldova. International Journal of Internet Technology and Secured Transactions, 7(2), 115-143. https://dx.doi.org/10.2139/ ssrn. 2732350

Ravello, J. (2012). 30 percent of aid lost to corruption - Ban Ki-moon, Blog: devex, 10 de julio del 2012. Recuperado de http://bit.ly/2Jn6kzP [Fecha de consulta: 15 de febrero de 2019]

Sharpley, R., \& Naidoo, P. (2010). Tourism and Poverty Reduction: The Case of Mauritius. Tourismand Hospitality Planning \& Development, 7(2), 145-162. https://doi.org/10.1080/14790531003737169 
Shen, D., Urquhart, A.,\& Wang, P. (2019). Does twitter predict Bitcoin? Economics Letters, 174, 118122. https://doi.org/10.1016/j.econlet.2018.11.007

Scheyvens, R. \& Hughes, E. (2018). Can Tourism help to "end poverty in all its forms everywhere"? The challenge of tourism addressing SDG1. Journal of Sustainable Tourism, 27(7), 10611079. https://doi.org/10.1080/09669582.2018.1551404

Snyman, S.L. (2012). The role of tourism employment in poverty reduction and community perceptions of conservation and tourism in southern Africa. Journal of Sustainable Tourism, 20(3),. 395-416. https://doi.org/10.1080/09669582.2012.657202

The World Bank (2017). Remittance prices worldwide: An analysis of trends in cost of remittance services. Recuperado de http://bit.ly/2JmskuR

Thomas, R., \& Koens, K. (2016). "You know that's a rip-off": policies and practices surroundings micro-enterprises and poverty alleviation in South African township tourism. Journal of Sustainable Tourism, 24(12), 1641-1654. https://doi.org/10.1080/09669582.2016.1145230

Thomason, J., Ahmad, M., Bronder, P., Hoyt, E., Pocock, S., Bouteloupe, J., ...\& Shrier. D. (2018). Blockchain-Powering and Empowering the Poor in Developing Countries. En A. Marke (Ed.), Transforming Climate Finance and Green Investment with Blockchains (pp. 137-152). London, UK: Elsevier.

Truong, V.D. (2018). Tourism, poverty alleviation and the informal economy: the street vendors of Hanoi, Vietnam. Tourism Recreation Research, 43(1), 52-67. https://doi.org/10.1080/02508 281.2017.1370568

Tu, K.V. (2018). Perfecting Bitcoin. Georgia Law Review, 52(2), 505-580.

Tucker, H., \& Boonabaana, B. (2012). A critical analysis of tourism, gender and poverty reduction. Journal of Sustainable Tourism, 20(3), 437-455. https://doi.org/10.1080/09669582.2011.6 22769

Tumusiime, D.M., \& Vedeld, P. (2012). False promise or False Premise? Using Tourism Revenue Sharing to Promote Conservation and Poverty Reduction in Uganda. Conservation and Society, 10(1), 15-28. https://doi.org/10.4103/0972-4923.92189

United Nations, Commission on Sustainable Development. (1999). El turismo y el desarrollo sostenible, 15 de enero de 1999. Recuperado de http://bit.ly/2JBawfm

Vincent, O., \& Evans, O. (2019). Can cryptocurrency, mobile phones, and internet herald sustainable financial sector development in emerging markets? Journal of Transnational Management, 24(3), 259-279. https://doi.org/10.1080/15475778.2019.1633170

World Travel \& Tourism Council (2019). Travel \& Tourism. Economic Impact 2019 World. Recuperado de http://bit.ly/2NY5Kg2

Yadav, M. (2017). Exploring Signals for Investing in an Initial Coin Offering (ICO), SSRN Electronic Journal. http://dx.doi.org/10.2139/ssrn.3037106

Yu, L., Wang, G., \& Marcouiller, D.W. (2019). A scientometric review of pro-poor tourism research: Visualization and analysis. Tourism Management Perspectives, 30, 75-88. https://doi. org/10.1016/j.tmp.2019.02.005

Zamani, E.D., \& Giaglis, G. M. (2018). With a little help from the miners: Distributed ledger technology and market disintermediation. Industrial Management $\mathcal{E}$ Data Systems, 118(3), 637-652. http://dx.doi.org/10.1108/IMDS-05-2017-0231

Zeng, B., Ryan, C., Cui, C., \& Chen, H. (2015). Tourism-generated Income Distribution in a Poor Rural Community: A Case Study from Shaanxi, China. Journal of China Tourism Research, 11(1), 85-104. https://doi.org/10.1080/19388160.2014.953281

Zharova, A., \& Lloyd, I. (2018). An examination of the experience of cryptocurrency use in Russia. In search of better practice. Computer Law and Security Review, 34(6), 1300-1313. https:// doi.org/10.1016/j.clsr.2018.09.004 